\title{
Integración, inclusión y justicia social; reflexiones desde las normativas inclusivas en la educación chilena
}

School integration, inclusion and social justice; thoughts from the inclusive norms in the chilean education system

\section{Volumen 20, Número 2 \\ Mayo - Agosto \\ pp. 1-28}

\author{
Paulina Véliz Jorquera \\ María Jesús Martínez \\ Hugo Parra Muñoz \\ Catalina Garrido Reyes
}

\section{Citar este documento según modelo APA}

Véliz Jorquera, Paulina., Martínez, María Jesús., Parra Muñoz, Hugo. y Garrido Reyes, Catalina. (2020). Integración, inclusión y justicia social: Reflexiones desde las normativas inclusivas en la educación chilena. Revista Actualidades Investigativas en Educación, 20(2), 1-28. Doi. 10.15517/aie.v20i2.41709 


\title{
Integración, inclusión y justicia social: Reflexiones desde las normativas inclusivas en la educación chilena
}

School integration, inclusion and social justice: Thoughts from the inclusive norms in the chilean education system

\author{
Paulina Véliz Jorquera ${ }^{1}$ \\ María Jesús Martínez ${ }^{2}$ \\ Hugo Parra Muñoz \\ Catalina Garrido Reyes ${ }^{4}$
}

\begin{abstract}
Resumen: Desde 2009, el sistema educacional chileno ha vivido una serie de ajustes y reformas estructurales tendientes a construir escuelas inclusivas que responden a las demandas sociales de equidad y calidad. Este proceso, con continuidades y rupturas, es abordado en este estudio para comprender los sentidos y alcances de lo que se ha comprendido por sistema educativo inclusivo orientado a la justicia social. En este contexto presentamos el despliegue analítico de una investigación cualitativa desarrollada entre los años 2017-2019 que cuestiona sobre los sentidos discursivos latentes en las políticas educativas en Chile en torno a la diversidad e inclusión en el sistema educacional. Se desarrolla una investigación política documental cualitativa, con un enfoque teórico hermenéutico con aspectos críticos. Este proceso investigativo ha encontrado que las configuraciones y reestructuraciones -a nivel paradigmático y social- que han emergido desde las políticas educativas responden a una fuente integradora y no a una matriz inclusiva; esto bajo una declaración discursiva relativa a la intención de entregar servicios educacionales de calidad y equitativos a la población escolar, que presenten o no condición de discapacidad. Otro resultado destacado en este artículo hace referencia a los cambios emergentes que se observaron durante el tránsito desde el enfoque integrativo al inclusivo; aspectos como los modos de materialización del discurso inclusivo en las aulas regulares, aquellos que proponen la diversificación de enseñanza y la eliminación de barreras frente al aprendizaje. Finalmente, el artículo reflexiona sobre el sustento y el análisis del recorrido de las políticas públicas educativas de carácter inclusivo que se han promulgado en la última década en Chile, declarando los silencios y contradicciones entre ellas.
\end{abstract}

Palabras clave: educación inclusiva, necesidades educativas especiales, políticas públicas, justicia social

${ }^{1}$ Universidad Tecnológica de Chile INACAP, Chile. Coordinadora y académica de la carrera de psicopedagogía. Dirección electrónica: pveliz@inacap.cl Orcid https://orcid.org/0000$\underline{0001-6172-6233}$

2 Universidad Academia de Humanismo Cristiano, Chile. Licenciada en Ciencias de la Educación. Psicopedagoga y Educadora Diferencial. Dirección electrónica: mariajesus.martinezp@gmail.com Orcid https://orcid.org/0000-0003-3136-968X

${ }^{3}$ University of Bristol, Reino Unido. Dr@ Educación, University of Bristol. Dirección electrónica: hugo.parramunoz@bristol.ac.uk Orcid https://orcid.org/0000-0003-0901-3003

${ }^{4}$ Universidad Metropolitana de Ciencias de la Educación, Chile. Licenciada en Educación y Pedagogía en Educación Diferencial, Universidad Metropolitana de Ciencias de la Educación. Dirección electrónica: cata.garrido@gmail.com Orcid https://orcid.org/0000-0002-2916-520X

Artículo recibido: 16 de octubre, 2019

Enviado a corrección: 30 de marzo, 2020

Aprobado: 27 de abril, 2020

Los contenidos de este artículo están bajo una licencia Creative Commons 


\begin{abstract}
Since 2009 chilean school system has experienced a series of adjustment, and structural reforms which tend to the consecution of inclusive schools that respond to the social claims for equity and quality. This process presents continuities and ruptures that might be tackled to comprehending the senses and scopes of the understandings about inclusive educative system oriented to social justice. In this context, we present the analytical deployment of a qualitative research, developed in 2017-2019, that questions the latent discursive senses in educational policies in Chile around diversity and inclusion in the educational system. This was a qualitative documental policy inquiry, with a hermeneutic and critic theoretical frameworks. The study presents social configurations and re-structurations which would be produced by the educative policies. They represent the forces of the integrative scheme in the core of the discourses about inclusion. policies which narrates the delivery of equitative and high-quality educative services to students who present or not disabilities. another main finding is linked to the emergences that are observed in the transit from an integrative scheme to an inclusive one; aspects such as the modes of materialization of the inclusive discourse in the regular classroom, they posit the diversification on teaching and the elimination of any learning barrier, finally, the article reflect on the displacements of the chilean public policies in education on the last decade, declaring its silences and contradictions.
\end{abstract}

Key words: school integration, special education needs, public policies, social justice

\title{
1. Introducción
}

Como investigadores nos mueven los sujetos pedagógicos que habitan en los márgenes del sistema educativo chileno, las otredades excluidas parecen estar confinadas a un espacio en donde el sentido de la pedagogía se enfrenta a la industrialización y profesionalización de la enseñanza. Los contextos de extrema privatización (Verger, Moschetti, y Fontdevila, 2017), la crisis del sistema educativo público (Opertti y Magni, 2018), y la alta segregación producto de la inequidad (García-Huidobro y Bellei, 2006) han sido vastamente abordados por investigaciones. Chile, en el contexto latinoamericano, presenta estas dicotomías y particularidades que nos llevan a cuestionarnos respecto a lo que se entiende en el clamor social de equidad y calidad para todos.

De allí que nos preguntemos respecto al surgimiento de políticas públicas que respaldan el derecho a la integración y/o inclusión educativa, como son el Decreto $\mathrm{N}^{\circ} 170$, Decreto N83, la Ley 20.248 de Subvención Escolar Preferencial (Ministerio de Educación de Chile, 2008), la Ley General de Educación, entre otras, y aún no avanzamos en construir un sistema educativo que acepte a las diferencias.

A partir de aquella emergencia surgen cuestiones respecto a (1) ¿cuáles son los sentidos desde donde las políticas educativas en Chile han pensado la construcción de escuelas inclusivas?; (2) ¿Qué tensiones/adaptaciones emergen en la articulación de normativas inclusivas y la cultura de resultado del sistema chileno?; (3) ¿De qué manera se relacionan los sentidos sobre la diferencia con aquellas personas que representan al otro?

A partir del problema e interés propuesto anteriormente, desarrollamos una investigación, entre los años 2017 y 2019, que buscaba reflexionar y profundizar en los 
sentidos discursivos latentes en las normativas chilenas -desde 1990 a 2018- que regulan la atención a la diversidad en el sistema educacional público, esas otredades sujetas a procesos inclusivos.

En este artículo mostramos el desarrollo analítico del conjunto de normativas relativas a la construcción de escuelas inclusivas, el que sugiere en ellas una práctica discursiva que oferta la provisión de oportunidades de educación equitativas y de calidad para todo el estudiantado.

A partir de lo reseñado, nuestros objetivos investigativos fueron: (1) analizar las nociones desde donde se ha entendido el proceso inclusivo descrito en las políticas educativas chilenas; (2) criticar la relación entre las lógicas de rendición de cuentas y los procesos inclusivos que configuran las normativas educativas chilenas; (3) valorar los entendidos sobre las otredades que son receptoras de los afanes inclusivos.

Estos objetivos nos guían al estudio de la fuerza de las normativas educativas asociadas al proceso inclusivo en Chile, en tanto políticas que trazan fronteras y definiciones de lo que se entiende por diversidad, inclusión, junto con operativizar las acciones que los establecimientos educacionales desarrollan sobre estos; pero, que silenciarían consideraciones sobre justicia social. Este ensamble de normativas sugiere a los Programas de Subvención Escolar Preferencial (Ministerio de Educación de Chile, 2008) y a los Programas de Integración Escolar (PIE) (Ministerio de Educación de Chile, 2009) como las respuestas del sistema educativo para la constitución de escuelas inclusivas y por tanto ejes centrales de nuestra investigación. En ella focalizamos nuestra atención en los PIE, analizando las dimensiones y sentidos con las que estas conceptualizaciones se encuentran vinculadas, puesto que dicho programa es el que directamente atiende en forma pedagógica, con incidencia curricular y evaluativa, a estudiantes que experiencian barreras para el aprendizaje.

EI PIE tiene presencia desde inicios de la década de 1990 con la promulgación del Decreto № 490 (Ministerio de Educación de Chile, 1990) que fija las primeras normativas que regulaban su implementación y la respectiva subvención por cada niño y niña participante. No obstante, es en 1998, con la promulgación de los Decretos Supremos № 01 (Ministerio de Educación de Chile, 1998) y 374 (Ministerio de Educación de Chile, 1999) cuando el PIE se instala como una estrategia relevante dentro del sistema educativo "para la integración social de personas con discapacidad" (Ministerio de Educación de Chile, 1998, p.1) a través de equipos multiprofesionales, adecuaciones curriculares y sistematizaciones 
administrativas, junto a aquello operativiza un proceso de certificación diagnóstica que permitiría impetrar subvención. Es importante señalar que en 2005 aparece la Nueva Política de Educación Especial (Ministerio de Educación de Chile, 2005), esta nueva perspectiva política declara una búsqueda de mayores oportunidades de acceso de estudiantes -que se enfrentaban a barreras para el aprendizaje- a los establecimientos de educación regular, además propone la revisión de instancias curriculares y administrativas, junto a aquello promueve la participación de otros actores de la comunidad educativa, acompañado del incremento de la subvención.

Los procesos de integración delimitados por las normativas educativas chilenas declaran, reconocen y definen la promoción de la aceptación de personas con discapacidad en las escuelas regulares; para, de esta manera, entregar un servicio educativo que se rija por los principios de universalidad, equidad y calidad en la educación para todos, tal como estipula la Ley General de Educación, en adelante LGE, (Ley N²0.370, Ministerio de Educación, 2009). Es necesario recordar que esta nueva normativa educativa nacional tiene su origen en los movimientos sociales, desde el mochilazo ${ }^{5}$ en 2004 , pero ya con más fuerza en la llamada revolución pingüina ${ }^{6}$ en el año 2006, que coincidían en la búsqueda de un mismo fin: educación gratuita y de calidad para todos, ya que entendían que la educación no es un bien de mercado, sino un derecho para la ciudadanía (Mayol y Azócar, 2011). Estos movimientos sociales promueven distintos cambios legales que ha sufrido el sistema educativo, que surgirían entonces, como respuesta a los clamores por la consecución de una sociedad más justa. Es así como, en el año 2009, se promulga la LGE, la cual regula tanto los derechos como los deberes de las comunidades educativas; respecto a lo mencionado resulta necesario destacar ciertos artículos que apuntan en su totalidad a la educación especial. Entre ellos se encuentra el artículo $3^{\circ}$, el cual ratifica el derecho a la enseñanza, señalando para esto a diversos principios que sostendrían al sistema educativo. Como principio relevante se destaca el de equidad, el cual "propenderá a asegurar que todos los estudiantes tengan las mismas oportunidades de recibir una educación de calidad, con

${ }^{5}$ La primera manifestación organizada por el nuevo organismo, que en su recorrido llegó hasta el centro de Santiago, se caracterizó por actos de vandalismo y por una fuerte represión policial (500 estudiantes fueron detenidos). Sucesivas protestas despertaron el interés de la prensa que, con motivo de ello, acuñó la palabra "mochilazo" (Borri, 2016).

6 La Revolución Pingüina fue como finalmente se terminó denominando al episodio de protesta estudiantil secundaria del año 2006 en Chile. El apodo con que se bautiza a los secundarios tiene un origen anecdótico. Justamente el año 2006 se estrena el documental "La Marcha de los Pingüinos" que muestra el camino que siguen los pingüinos de la Antártica a la tierra de gestación. La marcha de los pingüinos de la Antártica se asemeja a la marcha de los secundarios en las calles y el apodo "pingüino" hace alusión al color de los uniformes que ocupan los estudiantes (blanco y azul oscuro) (Silva, 2019). 
especial atención en aquellas personas o grupos que requieran apoyo especial" (Ley $\mathrm{N}^{\circ}$ 20.370, 2009).

Específicamente, hoy existen dos Decretos fundamentales que rigen a los PIE en los establecimientos educaciones chilenos, como respuesta a promesas existentes en la LGE (Ministerio de Educación de Chile, 2009) en relación a la atención educativa a la diversidad, como se observa en la vaguedad de su artículo 34:

En el caso de la educación especial o diferencial, corresponderá al Ministerio de Educación, previa aprobación del Consejo Nacional de Educación conforme al procedimiento establecido en el artículo 53, definir criterios y orientaciones para diagnosticar a los alumnos que presenten necesidades educativas especiales, así como criterios y orientaciones de adecuación curricular que permitan a los establecimientos educacionales planificar propuestas educativas pertinentes y de calidad para estos alumnos, sea que estudien en escuelas especiales o en establecimientos de la educación regular bajo la modalidad de educación especial en programas de integración.

Son ambos Decretos, 170 y 83, los que vendrían a complementar y llenar esos vacíos para los procesos educativos inclusivos. Por una parte el Decreto $N^{\circ} 170$ (Ministerio de Educación de Chile, 2009), fija normas para determinar los y las alumnos/as con Necesidades Educativas Especiales (NEE) que serán beneficiarios de las subvenciones para educación especial, con lo que se convierte en el aparato regulador que determina la gestión administrativa y clínica/pedagógica del funcionamiento de los PIE, estableciendo criterios de diagnóstico de las NEE, clasificando las NEE en transitorias y permanentes, limitando los cupos de ingreso de estudiantes al PIE y sugiriendo una estadía de aproximadamente 2 años para que estudiantes con NEE de carácter transitorias adquieran herramientas que le permitan enfrentar el proceso escolar de manera efectiva (con posibilidad de renovar el cupo, si es necesario), entre otros aspectos.

En tanto el Decreto 83 (Ministerio de Educación de Chile, 2015a) aprueba criterios y orientaciones de adecuación curricular para estudiantes con necesidades educativas especiales solamente para los niveles de educación parvularia y educación básica, teniendo como principios orientadores la Igualdad de oportunidades, Calidad educativa con equidad, Inclusión Educativa y Valoración de la diversidad y Flexibilidad en la respuesta educativa, proponiendo como estrategia concreta el Diseño Universal de Aprendizaje DUA (CAST, 
2008), con el fin de maximizar las oportunidades de aprendizaje del estudiantado. Este decreto define las adecuaciones curriculares como "una herramienta importante que permite acceder a los objetivos generales del currículum que señala la ley, en condiciones similares a las que acceden estudiantes sin necesidades educativas especiales" (Ministerio de Educación, 2015a, p.17). En otras palabras, se produce un movimiento reduccionista al sólo diversificar el acceso al currículum (los espacios de mediación docente), pero no la alteración del diseño curricular; se proyecta entonces que, el realizar estas adecuaciones permitiría que toda persona acceda al currículum según sus propias capacidades; si bien se plantea que las adecuaciones curriculares se despliegan, principalmente, en el aula regular en beneficio del grupo curso, se enfatiza al grupo de estudiantes que presenten Necesidad Educativa Especial, ejecutando una discriminación positiva.

Estos Decretos conforman administrativamente a los PIE como el aparato que ofrecería estrategias inclusivas en el sistema escolar, que entregaría oportunidades de aprendizaje diversificadas, de modo que toda persona pudiese progresar en sus aprendizajes, en especial los que presente Necesidades Educativas Especiales.

Teniendo a la vista esta normativa que determina y subvenciona sólo ciertos tipos de diferencias -asociadas a procesos de certificación diagnóstica/clínica de las NEE- para construir una educación de calidad para todos, es lo que nos lleva a creer necesario analizar las trayectorias por las que los procesos integrativos-inclusivos han atravesado, desplazamientos que podrían sugerir intereses e intencionalidades de los cambios que han sufrido estos procesos de apertura del sistema educativo.

\section{Encuadre teórico conceptual}

Como señalamos en la sección anterior, la necesidad de integración educativa surge a partir de las prácticas de exclusión que la sociedad en su conjunto ha efectuado sobre las personas que se encuentran fuera de las normas establecidas por una minoría. En ese contexto queremos hacer referencia a lo que expresa Echeita (2008),

(...) no pretendo caer en la ingenuidad de pensar que la educación escolar es la puerta más importante para paliar los procesos de exclusión social, ni en los casos de extrema pobreza, ni en otros menos dramáticos (...), pero es una de ellas, y nuestra responsabilidad como educadores es que lo que hagamos puertas adentro de la escuela sea parte de la solución y no parte del problema. (p.10) 
La exclusión, es lo que intentan eliminar las instituciones educativas para generar espacios de igualdad, pero en este proceso el sistema educativo chileno ha carecido de debates y diálogos sociales respecto a lo que implica el concepto de "igualdad" en contextos de una Sociedad de matriz económica distributiva no equitativa, las implicancias de estos contextos olvida reflexionar sobre los procesos de justicia social necesarios para la construcción de una sociedad que oferte igualdad de oportunidades -en este caso de aprendizajes- para el alumnado que se han visto en desmedro y desventaja; y, de este modo, lograr derribar aquellas barreras que impiden una educación justa e igualitaria para todos; como bien señala Echeita (2008), no podemos reducir la construcción de sociedades más equitativas pensando en solo intervenir en las prácticas de enseñanza del profesorado. Se debe avanzar hacia reestructuraciones societales (Fraser, 1997) respecto a las alteridades en el aula, que primero pasa por un reconocimiento del otro, no desde la perspectiva de un igual que finaliza por positivar la diferencia (Parra, 2016).

El reconocimiento es una necesidad vital humana, con dos tipos: igual dignidad para todos, y la política de la diferencia, donde cada uno es reconocido por su particular identidad. Por tanto, se demanda ser universalmente iguales, en derechos y libertades. Esta petición de igual reconocimiento se extiende más allá de la declaración del igual valor que todos los humanos potencialmente tenemos para convertirlo en un hecho real. (Murillo y Hernández, 2011, p. 20)

Desde esa perspectiva, las normativas educativas de intenciones inclusivas pretendería la constitución, desde el espacio escolar, de una sociedad más justa, que permita entregar educación a la totalidad del estudiantado, pero comprendiendo esas diversidades desde sus condiciones físicas, mentales y/o económicas; suprimiendo las diferencias de las complejidades subjetivas que también existen y basando esa mirada solo en estudiantes, no reconociendo al cuerpo docente y administrativo como fuentes de diferencias.

En la política educativa revisada, no destaca una búsqueda de Justicia Social, la intenta otorgar igualdad de derecho para todos, en los distintos escenarios que pretendemos estar de protagonistas para ser un aporte dentro de la sociedad, no solo basta con una compensación económica igualitaria, sino más bien, al acceso a la educación y a la participación (Murillo y Hernández, 20011), pero que debe avanzar a procesos de reestructuración de las culturas sociales respecto a las alteridades (Fraser, 2008; Diez, 
2004). En ese escenario debemos reconocer que se ha intentado la construcción de escuelas abiertas a la participación de quienes han sido excluidos, pero que se ha omitido cualquier tipo de consideración relativa a las implicancias de construir una sociedad más justa. Las nuevas normativas chilenas sustentan cambios significativos, en términos estructurales de gestión y de diseño curricular de aula, pero no suficientes para lograr la inclusión de toda la comunidad estudiantil.

Uno de los cambios más relevantes de los últimos años es el Programa de Integración Escolar (PIE) donde el Ministerio de Educación pretende que los establecimientos educaciones tomen la iniciativa de incorporarlos para poder integrar a sus sistemas a niños y niñas con Necesidades Educativas Especiales, orientados por el enfoque inclusivo definido por UNESCO (MINEDUC, 2013), que señala "EI PIE es una estrategia inclusiva del sistema escolar cuyo propósito es entregar apoyos adicionales, en el contexto del aula común, a estudiantes que presentan Necesidades Educativas Especiales (NEE)" (p. 7), en relación a lo cual habría que preguntarse: ¿Por qué, a pesar de avanzar a lógicas inclusivas, la normativa se mantiene en posicionar al alumnado como poseedores de cierta carencia en su condición de sujeto incluido, no habiendo una transformación a que aquella condición que sitúa al sujeto como incluido es producida e instalada por los contextos educativos, sociales y culturales?.

Luego detalla: “...sean éstas de carácter permanente o transitorio, favoreciendo la presencia y participación en la sala de clases..." (р. 7). Frente a esto es necesario que nos preguntemos ¿La mera Presencia y participación aseguran la aceptación del otro como un sujeto en igualdad de condiciones? Ante esto, los análisis nos sugieren que esta condición de equidad se da cuando entregamos al otro una condición de capacidad de decidir sobre sus propios procesos, es decir reestructurando las políticas internas del aula y la escuela.

Si continuamos con el análisis de este documento, vemos que en este se propone “...el logro de los objetivos de aprendizaje" (p. 7), por lo que queda eminentemente manifestado la orientación del proceso educativo inclusivo en la rendición de cuentas. En este punto es importante señalar que la idea del mejoramiento continuo, propio de las lógicas de la nueva Gestión Pública, es parte de la racionalidad neoliberal (Han, 2017) que estandariza a los sujetos, lo que queda estipulado cuando el documento expresa "...la trayectoria educativa de 'todos y cada uno de los estudiantes', contribuyendo con ello al mejoramiento continuo" (p. 7). En este mismo texto se refuerza este vínculo enseñanzaeficiencia-aprendizaje planteando respecto a la calidad del servicio educativo "alinear todos 
los esfuerzos en la dirección del mejoramiento educativo y de conseguir los mejores resultados de aprendizaje" (p. 7). Sobre este punto surgen cuestiones sobre ¿qué entiende el MINEDUC por mejores resultados de aprendizaje?, ¿sólo aquellos que el Consejo Nacional de Educación estandarizó?; y, ¿Quién determina lo que sería mejor y para qué lo estaría determinando?

De esta forma, se desprende una contradicción cuando desde la política pública se habla de inclusión y se diseña un programa con las características descritas en el de Integración Escolar, quedando a la vista una ambigüedad sobre la semántica de significados de dichos conceptos, relegando la condición de estrategia inclusiva a un mero intento teórico de inclusión.

Hemos comprendido la conceptualización de educación inclusiva como finalidad de todo aprendizaje grupal e individual en un establecimiento educativo, independiente de las Necesidades Educativas Especiales -ya sean las certificadas por el Decreto 170 o las de las diversidades que éste no considera- que presente el estudiantado. De esta manera, a diferencia de lo que plantea MINEDUC y UNICEF una enseñanza inclusiva no sería una búsqueda permanente si desde su fuente -el entramado subjetivo del cuerpo docente - no se haya instalada una concepción de aceptación a las diferencias, y además esta vaguedad definitoria de inclusión como proceso de "búsqueda permanente", que no establece en claridad aquello que se debe buscar, oscurece los avances inclusivos en las escuelas, que para nosotros son inherentes al desarrollo de una educación socialmente justa.

Pues bien, el MINEDUC entiende la inclusión como herramienta de acceso de personas con algún tipo de Necesidad Educativa Especial a los establecimientos educativos regulares (acotándola a aspectos de la arquitectura educativa), aspirando a eliminar las barreras que delimitarían sus aprendizajes (reduciéndola a estrategias didácticas) y promoviendo la activa colaboración del estudiantado, en un Sistema educativo que tiende a los procesos de individualización y competencia (Ministerio de Educacion, 2015a). Cabe destacar la importancia que el sistema educativo entrega a la transformación estructural de los colegios desde la liberación de los proyectos educativos que se expresan en misiones y visiones, pero que no necesariamente implican reestructuraciones societales sobre las diferencias ni menos alcanzan las practicas pedagógicas del establecimiento académico. De esta manera, el acto inclusivo queda confinado a ser un acto discursivo que se propone realizar cambios. 
Para complementar con lo anterior, es importante mencionar que las conceptualizaciones que se entienden sobre una escuela inclusiva buscan que la totalidad de la comunidad estudiantil reciban recursos y procesos de enseñanza adaptados a sus necesidades, no solo aquellos que presentan Necesidades Educativas Especiales (Parra, 2010). Si bien existen colegios que integran a estudiantes con discapacidad, no todos acceden a entregar una enseñanza significativa y adaptada a sus propios procesos de aprendizaje. Por el contrario, estudiantes con Necesidades Educativas Especiales deben adaptarse a las enseñanzas del profesorado para lograr aprender lo que se entrega en las salas de clases, es por esto que "En este modelo se considera que es de responsabilidad de las personas con discapacidad lograr por sí mismos su plena integración, correspondiente al modelo de normalización, distinto al actual modelo de enfoque social de la discapacidad" (Parra, 2010, p. 82). Siendo este acto de "superación de las condiciones personales" parte de la narrativa meritocrática del Sistema económico imperante (Khan, 2011; Piketty, 2013).

Es por este motivo que se apunta a las escuelas inclusivas donde el profesorado debe entregar una enseñanza diversificada para que todos accedan al aprendizaje, es decir, "la educación inclusiva debe ser 'proactiva' en la identificación de barreras que algunos grupos encuentran al intentar acceder a las oportunidades educativas" (Parra, 2010, p. 83). Quedando reducida a los campos de acción del cuerpo docente, olvidando que éstos son sujetos socialmente construidos y que portan en sus subjetividades las cargas totalizadoras y estandarizantes de negación a las diferencias -incluso de ellos mismos-.

A través del PIE se busca contribuir al mejoramiento continuo de la calidad de la educación en el establecimiento educacional favoreciendo, en el ámbito curricular, el desarrollo e implementación de estrategias diversificadas, basadas en el Diseño Universal del Aprendizaje (DUA), que permite dar respuestas educativas ajustadas a las necesidades y características de todos los alumnos y alumnas, no sólo de los que presentan NEE. Además, constituye una herramienta para mejorar las relaciones interpersonales y el clima de convivencia escolar entre los estudiantes y distintos actores de la comunidad educativa. (MINEDUC, 2013, p. 8)

\section{Metodología}

\subsection{Enfoque}

La investigación se desarrolló durante los años 2017-2018, se sustenta en una mirada cualitativa del fenómeno inclusive en el Sistema educativo, contempla un desarrollo 
hermenéutico (Ricoeur, 2000; Valverde, 2003). con aspectos críticos, definido como "la teoría de las reglas que gobiernan una exégesis, es decir, una interpretación de un texto particular o colección de signos susceptible de ser considerada como un texto" (Ricoeur en Sandoval, 2002, p.67). Dicho enfoque fue escogido, debido a que propone una interpretación de la realidad social, cimentado en el análisis crítico de las diversas normativas a analizar, para que de esta forma incorporar las diversas dimensiones en las cuales se fundamenta nuestro estudio. Por su parte, el enfoque cualitativo evalúa el proceso natural de los hechos, no existiendo una manipulación de la realidad, basándose en una interpretación de los datos obtenidos de ésta. de esta manera resulta evidente que presentamos una investigación cualitativa (Flick, 2004), descriptiva y de diseño emergente (Ritchie y Lewis, 2003).

\subsection{Unidades de análisis}

Para el desarrollo de esta investigación se analizaron 10 documentos legales, promulgados desde el año 1990 hasta 2018 (Tabla 1). Se considera dicho periodo por que marca el cambio político social de la instalación de la cultura de rendición de cuentas en el sistema educativo, otro aspecto a considerar es que en 2009 se promulga la LGE que es la que redefine al sistema educativo e instala las racionalidades gerenciales en el sistema educativo chileno (Morales, 2014; Falabella, 2015).

Tabla 1. "Documentos legales relacionados con el sistema educativo seleccionados para análisis, según origen, año y criterio de inclusión, Chile 1990-2015"

\begin{tabular}{|c|c|c|c|c|}
\hline & Documento & Origen & Año & Criterio de inclusión / nombre \\
\hline 1 & Decreto Supremo № 490 & $\begin{array}{l}\text { Ministerio de } \\
\text { Educación de Chile. }\end{array}$ & 1990 & $\begin{array}{lcr}\text { Establece } & \text { normas para integrar } \\
\text { alumnos } & \text { discapacitados } & \text { en } \\
\text { establecimientos comunes. } & \\
\end{array}$ \\
\hline 2 & Decreto Supremo № 01 & $\begin{array}{l}\text { Ministerio de } \\
\text { Educación de Chile. }\end{array}$ & 1998 & $\begin{array}{l}\text { Reglamenta capítulo II título IV de la } \\
\text { Ley No } 19.284 \text { que establece normas } \\
\text { para la Integración Social de } \\
\text { Personas con Discapacidad. }\end{array}$ \\
\hline 3 & Decreto Supremo N 374 & $\begin{array}{l}\text { Ministerio de } \\
\text { Educación de Chile. }\end{array}$ & 1999 & $\begin{array}{l}\text { Complementa Decreto Supremo de } \\
\text { Educación № } 01 / 1998 \text {, autorizando } \\
\text { la atención de los escolares } \\
\text { hospitalizados. }\end{array}$ \\
\hline 4 & $\begin{array}{lr}\text { Ley } \quad \mathrm{N}^{\circ} & 20.248 \mathrm{de} \\
\text { Subvención } & \text { Escolar } \\
\text { Preferencial } & \end{array}$ & $\begin{array}{l}\text { Ministerio de } \\
\text { Educación de Chile. }\end{array}$ & 2008 & $\begin{array}{l}\text { Establece normas para entregar } \\
\text { recursos adicionales por cada } \\
\text { alumno prioritario y preferente, a los } \\
\text { sostenedores de establecimientos } \\
\text { que han firmado con el MINEDUC un } \\
\text { Convenio de lgualdad de } \\
\text { Oportunidades y Excelencia } \\
\text { Educativa; para la implementación de } \\
\text { un Plan de Mejoramiento educativo. }\end{array}$ \\
\hline
\end{tabular}




\begin{tabular}{|c|c|c|c|c|}
\hline 5 & $\begin{array}{lcc}\text { Ley } & \text { General } & \text { de } \\
\text { Educación No20.370 } & \\
\end{array}$ & $\begin{array}{l}\text { Ministerio de } \\
\text { Educación de Chile. }\end{array}$ & 2009 & $\begin{array}{l}\text { Esta Ley determina las bases de la } \\
\text { educación chilena. }\end{array}$ \\
\hline 6 & $\begin{array}{l}\text { Ley de Inclusión Social } \\
N^{\circ} 20.422\end{array}$ & $\begin{array}{l}\text { Ministerios de } \\
\text { Desarrollo Social y } \\
\text { Familia de Chile. }\end{array}$ & 2010 & $\begin{array}{l}\text { Establece Normas sobre lgualdad de } \\
\text { Oportunidades e Inclusión Social de } \\
\text { Personas con Discapacidad y } \\
\text { determina los principios de vida } \\
\text { independiente, accesibilidad } \\
\text { universal, diseño universal, } \\
\text { intersectorialidad, participación y } \\
\text { diálogo social. }\end{array}$ \\
\hline 7 & Decreto Supremo N¹70 & $\begin{array}{l}\text { Ministerio de } \\
\text { Educación de Chile. }\end{array}$ & 2010 & $\begin{array}{l}\text { Establece normas para determinar e } \\
\text { integrar a los alumnos con } \\
\text { Necesidades } \\
\text { Educativas Especiales que serán } \\
\text { beneficiarios de las } \\
\text { subvenciones para educación } \\
\text { especial. }\end{array}$ \\
\hline 8 & Ley $N^{\circ} 20.609$ & $\begin{array}{l}\text { Ministerio Secretaría } \\
\text { General de Gobierno } \\
\text { de Chile. }\end{array}$ & 2012 & $\begin{array}{l}\text { Establece medidas contra la } \\
\text { discriminación. }\end{array}$ \\
\hline 9 & Ley $N^{\circ} 20.845$ & & 2015 & $\begin{array}{l}\text { Inclusión escolar que regula la } \\
\text { admisión de los y las estudiantes, } \\
\text { elimina el financiamiento compartido } \\
\text { y prohíbe el lucro en } \\
\text { establecimientos educacionales que } \\
\text { reciben aportes del estado. }\end{array}$ \\
\hline 10 & Decreto Supremo Nº 83 & $\begin{array}{l}\text { Ministerio de } \\
\text { Educación de Chile. }\end{array}$ & 2015 & $\begin{array}{l}\text { Este decreto determina los modos en } \\
\text { que el sistema educativo debe } \\
\text { atender a la diversidad presente en } \\
\text { aula. }\end{array}$ \\
\hline
\end{tabular}

Fuente: Elaboración propia, 2019

\subsection{Técnicas de recolección de información}

Debido a la extensión de artículos describiremos algunos elementos críticos de los instrumentos de recolección de información. Primero, siguiendo la lectura de Riscoeur (2000) y de en artículos referidos a los temas "educación inclusiva”, "integración e inclusión en chile", equidad y calidad en educación en chile" nos llevan a concluir que dichas normativas son las que configuran las características actuales del sistema educativo y trazan la trayectoria de sus procesos inclusivos, buscando eliminar cargas subjetivas que se portan respecto a ellos.

La búsqueda de esos artículos siguió los criterios de ser publicaciones cuya indexación fuese al menos SCOPUS (q3). los filtros utilizados fueron: artículos con referato externo, de 5 años de antigüedad, con temáticas claves relativas a: (1) escuelas inclusivas, (2) procesos de inclusión (3) políticas educativas (5) exclusión escolar/educativa (6) programas de integración. Todos esos criterios en el sistema educativo chileno. 
Un tercer elemento de búsqueda se desarrolló en la lectura de los resúmenes seleccionando aquellas publicaciones que hacían referencia al análisis de políticas públicas (análisis político del discurso, ensayos o monográficos) y no a desarrollos investigativos empíricos. ya que nuestras preguntas de investigación se orientan hacia la teorización de sentidos y significados, no a prácticas. Al finalizar la búsqueda se obtuvieron 25 artículos, los que fueron usados como primera aproximación a la problemática tratada y dieron forma a la determinación de los cuerpos legales ya presentados.

\subsection{Procesamiento de análisis}

Decidimos comenzar el análisis por año de publicación, desde más antiguo en adelante, se realizó un doble fichaje de los documentos, los que fueron debatidos por el equipo de investigación hasta lograr el consenso interpretativo. en este proceso de fichaje y análisis temático (glisczinski, 2018) se utilizó la herramienta NVIVO (v.11), para facilitar el trabajo colaborativo. se realizó un proceso de codificación temática que nos permitió crear espacios de inteligibilidad interpretativa y abrió horizontes a elementos críticos.

\section{Resultados}

Desde los documentos estudiados, hemos encontrado que la noción de inclusión se utilizó para que todos aquellos que siempre fueron excluidos en la educación regular aceptaran nuevas formas más sutiles de exclusión (Skliar, 2002), cooptando cualquier disidencia y, de esta forma, instalar la narrativa de una nueva terminología que buscaría brindar una mayor esperanza sobre su inclusión dentro de las escuelas. Parra (2010) señala que, al empezar a manejar conceptos de educación integrada, se generaban estas instancias más sutiles de exclusión como el nacimiento de las escuelas especiales, la pedagogía terapéutica y la categorización de las necesidades educativas, entre otros.

En otras palabras, es un discurso que coopta las disidencias y resistencias en un sistema educativo, bajo una sociedad de control disciplinario (Foucault, 2009), donde todos se mantendrían expectantes frente a este nuevo cambio a nivel social. Sin embargo, en Chile -como señala esta cita obtenida durante nuestro análisis- se comienza a ubicar/utilizar dentro de las políticas públicas esta terminología inclusiva, mas no se implementa como tal, desplazándola hacia lógicas integrativas (Parra, 2010). Su puesta en marcha suponía, en primera instancia, la sensibilización a la sociedad frente a este tema, para luego llegar a una real inclusión; pero, sobre todo, educando a los actores educativos sobre el tema para que 
sus prácticas pedagógicas fueran inclusivas, reduciendo de esta manera las responsabilidades de una reestructuración de la sociedad en su conjunto a las estrategias didácticas del profesorado.

En el ámbito educativo, el Diseño Universal se expresa como Diseño Universal de Aprendizaje (DUA), cuyos criterios buscan promover prácticas inclusivas constituyendo el primer paso para responder a las diferencias individuales en el aprendizaje que presentan los estudiantes. (Ministerio de Educación, Decreto № 83, 2015, p. 17)

Si bien Parra (2010) afirma que el alumnado de colegios inclusivos, se benefician de adquirir aprendizajes adaptados a sus propios ritmos, es importante preguntarse qué ocurre con los establecimientos educacionales que presentan Programa de Integración Escolar y que no utilizan ni practican la terminología de la inclusión educativa: ¿efectivamente el profesorado adapta su enseñanza a la diversidad en la comunidad estudiantil que forma parte de su sala de clase? Para complementar esto, Carlos Skliar en la conferencia Estar Juntos en la Escuela (2014), nos habla de la importancia de estar y aprender juntos en las aulas y sobre el rol del profesorado para lograr una real inclusión educativa:

Nos seguimos preguntando sobre esa presencia, cuando en realidad, creo que hoy no es tan interesante preguntarnos por esa presencia, sino por algo que llamaremos y trataremos de trabajar y aclarar y pensar, la existencia de los demás. Estar presente es ni más ni menos que una posición material, es cierto que antes ni siquiera muchos no estaban presentes, pero una vez que estamos presente. Cuánto los proyectos educativos tocan la existencia de la gente, cuánto se vuelven fundamentales para su existencia, cuánto de su existencia es conocida por los maestros, cuánto de su existencia pesa a la hora de planificar, de pensar, de proponer actividades. (Skliar, $2014,4: 33)$

En relación con esto, si el Ministerio de Educación (2015b) sugiere que la construcción de estos Proyectos Educativos Institucionales (PEI) se realicen en instancias participativas, no es un requisito, y solo se realiza de manera informativa a toda la comunidad escolar, limitando las posibilidades de una verdadera inclusión. Ante esto, Ascorra, López, y Urbina (2016) indican que más bien se trata de una pseudo participación, es decir, que la escuela que parece participativa, en el fondo, presenta espacios de participación que están concretamente delimitados sin existir participación democrática real. 
De esta manera, podemos pensar en la forma en que las instituciones educativas toman la relevancia necesaria y el significado semántico que representa la inclusión educativa, puesto que se cree que tan solo con aceptar a estudiantes dentro de las aulas están cumpliendo con esta terminología, no tomando conciencia de la real transcendencia y de la concepción de esta misma. En otras palabras, ¿cómo podemos considerar la constitución actual de las escuelas como inclusivas si por Ley se encuentran limitadas a aceptar, entender e incluir las tipologías de las diferencias que certifica el Decreto $\mathrm{N}^{\circ} 170$ ? Aquí se consideran las Necesidades Educativas Especiales transitorias, como Trastornos Específicos del Aprendizaje, Trastornos Específicos del Lenguaje, Trastorno Déficit Atención con y sin hiperactividad o Trastorno Hipercinético, y rendimiento en prueba de coeficiente intelectual limítrofe; y de carácter permanente. Para el sistema educativo chileno, solo esas son las dificultades presentes y reconocidas en las aulas.

De igual modo, es importante mencionar que el desplazamiento del sentido de lo que conlleva la palabra inclusión es netamente político, dado que el estado requiere de cumplir con estándares internacionales que exigen cambios a nivel educativo y social, debido a los intereses como el reconocimiento internacional enfocado en la economía, de allí la importancia que cobran los informes OCDE que finalmente son los que impulsan los procesos de reforma y no los clamores del malestar de la ciudadanía (Mayol y Azócar, 2011).

Se deja entrever que, a pesar de las intenciones discursivas de las autoridades por incluir a estudiantes con o sin Necesidades Educativas Especiales, esto colisiona con las lógicas de la rendición de cuentas del aprendizaje y la estandarización de la evaluación, tensión observable en la presión de cobertura curricular sobre el cuerpo docente que obliga a la estandarización y lleva a profesorado y estudiantes a la exhaustación. estos procesos de estandarización se contradicen con las concepciones planteadas respecto a la justicia social, de este modo se confirma lo que Gabriel Salazar señala respecto a la estratificación y composición de clase del sistema educativo chileno (Pairo, 2011), donde unos pocos se benefician del aprendizaje y muchos otros se ven delimitados por la falta de una planificación diversificada, donde las clases o proyectos educativos no son pensados para ellos, viviendo nuevamente la exclusión dentro de la educación.

Para complementar, queremos exponer un escrito de Infante (2010) donde aporta a lo mencionado anteriormente, relacionando la inclusión con un otro que no ha tenido la opción de ser participe en la educación regular. 
Así, el concepto de inclusión hace referencia a la construcción de un 'Otro' (alumno que sale de los límites de la norma), a uno que no ha tenido el privilegio o, en palabras actuales, el derecho de estar en esos espacios educativos. Se busca, por tanto, aproximar a esos Otros a estos espacios regulares construidos social y culturalmente como centros. (Infante, 2010, p. 289)

En la actualidad, se propone discursivamente incluir en las aulas regulares a la diversidad de estudiantes que no han podido ser parte de ellas debido a la exclusión educativa, que ha llevado años segregando a ese otro que no se encuentra dentro de la norma para los parámetros educativos. La inclusión tiene como finalidad hacer partícipe a todos esos otros, para que tengan acceso a la educación y aprendizaje de manera igualitaria, cosa que por cierto se encontraría lejos de ser concretado en la realidad de las aulas educativas chilenas. Infante (2010) plantea que la inclusión es la construcción de un otro, ese otro es aquel estudiante que ha sido segregado durante décadas en las aulas regulares, ¿acaso ese otro no está construido? ¿pero ese otro en una sala es sólo uno, o la otredad implica la multivocidad de sujetos?

Nos es relevante destacar la mirada educativa desde donde se levantan los discursos inclusivos respecto a las otredades que son objeto del proceso inclusivo, las normativas entienden que las personas con discapacidad no están construidas y la inclusión viene a ayudarlos a completarse para poder ser asimilados como fuerza de trabajo (Ley $\mathrm{N}^{\circ} 20.370$, 2010). Sin embargo, esto se contrapone de sobremanera a lo que se entiende por inclusión, puesto que se debe aceptar a la diversidad que se encuentra en la comunidad estudiantil con sus diferencias, para comenzar a trabajar un proceso de enseñanza-aprendizaje, con la finalidad de respetar sus cualidades y ritmos de aprendizajes. Otro punto en el que se contrapone a la inclusión es que plantea una aproximación del otro en los espacios educativos, pero ¿solo basta con una aproximación? La inclusión va más allá que una simple aproximación: es estar dentro de aquellos espacios, al igual que el resto del alumnado, respetando cada una de las diferencias, siendo validado en su voz, en su capacidad de decisión y participación, siendo valorado como portador de conocimiento y cultura.

Para contraponer lo expuesto en la cita anterior, "Si se analiza el término inclusión desde la perspectiva educativa, es hacer efectivo para todos, el derecho a la educación, contemplando la igualdad de oportunidades, la eliminación de barreras para el aprendizaje y la participación en el contexto físico y social" (Parra, 2010, p. 81). A partir de esto podemos 
cuestionarnos cuántos niños y niñas que presentan diversas necesidades educativas no tienen respuesta a sus propias necesidades para lograr una educación igualitaria y, de esta manera, superar las barreras de aprendizaje que los limitan para aprender. La Ley de Subvención Escolar Preferencial (Ley SEP), a través del DFL Nº 2 de 1998 del Ministerio de Educación, establece que "de acuerdo a las necesidades educativas especiales de dichos alumnos deban ser atendidos en cursos de no más de 8 estudiantes..." (Ley 20.201, 2007), y que resultan insuficientes para dar respuesta a todas las necesidades de sus estudiantes, ya sean necesidades educativas transitorias o permanentes (como acceso o desarrollo de una vida escolar no diferenciada) (Peña, 2013).

Si bien hay ciertos establecimientos que sí han logrado romper estas barreras, son la minoría, ya muchos miembros del estudiantado continúan con sus limitaciones en el acceso al aprendizaje, desertando a la educación o pasan toda la vida cambiándose de establecimientos educacionales para lograr encontrar la escuela en la que ellos se puedan adaptar (López, 2014). De esto se infiere que, el sistema educativo en su conjunto sigue en una lógica de la medianía, de estandarizar y ejecutar sus acciones a partir del sujetoestudiante idealizado, normalizado, desde la totalización de la mismidad (Skliar, 2002). La inclusión habla de todo lo contrario, pues los colegios deben incluir con todo lo que implica la palabra a sus estudiantes y trabajar para que cada uno de ellos tenga acceso al aprendizaje de forma igualitaria y de equidad, independiente de sus necesidades y/o diferencias.

Sin lugar a duda, la inclusión va más allá que integrar al estudiantado que experimenta alguna de las Necesidades Educativas Especiales en los establecimientos y aulas regulares. La inclusión, es la forma de ver al otro como un igual, saber que no basta solo su presencia como lo menciona Skliar (2014), sino más bien, saber de la existencia del otro y como docentes tener la labor de enseñar a todos de acuerdo con su propia vida, procesos, intereses, habilidades, capacidades, entre otras. Esto implica una esfera no abordada por las reformas educativas, los alcances subjetivos en el profesorado que se requieren para reestructurar la cultura escolar hacia las diferencias.

Como ejemplo de las dificultades contextuales que impiden al profesorado en pensar sobre sus estudiantes tenemos que, cuando se dictamina la coordinación del equipo PIE, el diseño del trabajo colaborativo curricular y evaluativo, se debe considerar lo que se determinan las Orientaciones Técnicas (Ministerio de Educación, 2013), las cuales son del año 2013 y no han sido actualizadas hasta el momento. Aquí es donde se denota la primera falencia, dado que como son orientaciones que emanan desde el Ministerio de Educación, 
dependerá de cada establecimiento si es que quiere seguirse al pie de la letra. Por ejemplo, las orientaciones detallan que se debiesen asignar 3 horas cronológicas para el cuerpo docente de educación regular para planificar, evaluar y realizar diversos procesos asociados a una mejora en la calidad de la educación, lo que no es cumplido a cabalidad por los establecimientos, dado que en ocasiones dichas horas son otorgadas a partir de las horas que ya tiene el profesorado de planificación, no logrando cumplir con los objetivos que se proponen de manera adecuada por falta de tiempo. A su vez, tal como lo mencionan las orientaciones técnicas, dichas 3 horas se deben distribuir de manera flexible, tanto entre el cuerpo docente con responsabilidades de jefatura o líder de grupo de clase, como en quienes no comparten esa responsabilidad, lo que no alcanza para ejecutar un trabajo colaborativo adecuado y que sea el objetivo principal realizar cambios significativos en temas de inclusión y de mejorar la calidad de la educación en los colegios.

Aportando al extracto anterior, Parra (2010) habla del real sentido que tiene la inclusión y qué tipo de respuesta se debe entregar a quienes siempre han sido excluidos en los espacios educativos:

El verdadero sentido radica en brindar respuestas educativas que aseguren el derecho a acceder a la educación para todos los estudiantes, de manera equitativa, de acuerdo a sus características y dificultades individuales, poniendo énfasis en aquellos grupos o colectivos que siempre fueron excluidos del sistema educativo general. (p. 82)

El Ministerio de Educación propende a ofrecer al estudiantado la posibilidad de desarrollarse como personas libres, con conciencia de su propia dignidad y como sujetos de derechos, y contribuir a que el estudiantado tenga la oportunidad de desarrollar plenamente su potencial, independientemente de sus condiciones y circunstancias de vida. Ello supone tener presente la diversidad en los aspectos físicos, psíquicos, sociales y culturales propios de la naturaleza humana (MINEDUC, 2015a, p.12).

Sin embargo, investigaciones señalan que, en la política educativa, se ha producido un detrimento de la calidad con equidad mediante la implementación de sistemas de rendición de cuentas de los establecimientos basados en el rendimiento académico de sus estudiantes (Ascorra, Carrasco, López, y Morales, 2019). Sisto (2012) afirma que el trabajo docente se encuentra tensionado por su contribución social en el ámbito de la educación, y el trabajo docente en contextos de implementación de políticas educativas basadas en evaluación e incentivos al desempeño. 
La revisión de la documentación sugiere que, luego que la integración tomó el espacio suficiente dentro de los discursos políticos sobre educación, se comenzó a percibir que el alumnado que experiencia necesidades educativas especiales solo ocupaba un espacio dentro de las aulas, teniendo que adaptarse a las metodologías, currículum y evaluaciones que el establecimiento educacional les exigía (Skliar, 2012), y que la responsabilidad de las dificultades es individual, propia del estudiantado, y no del sistema educativo que se adapte a ellos (Inostroza, 2015; López, 2014), trayendo consigo consecuencias como volver a escuelas diferenciales o simplemente luchar día a día para lograr ser parte de la comunidad educativa.

La inclusión viene a dar respuesta a todo lo que quedó inconcluso con la integración y, por este motivo, creemos que es necesaria para remediar la situación descrita, pero que, mientras esta no sea asociada a la concepción de justicia social quedará relegada a aspectos superficiales en su instalación. Cada vez se observan más escuelas con proyectos e intenciones inclusivas, muchos se encuentran trabajando para mejorar sus prácticas pedagógicas con la finalidad de lograr entregar una enseñanza diversificada, eliminando las barreras de aprendizajes dentro de las salas de clases y hacer que todo estudiante aprenda, respetando sus ritmos y estilos propios de aprender. Sin embargo, esos proyectos se mantienen bajo la lógica neoliberal de la meritocracia y el entendimiento de la escuela como espacio de producción de la futura fuerza laboral. Queda mucho por hacer y aprender como sociedad, no solo basta que las comunidades educativas se empapen en conocimiento sobre la inclusión - a través de las capacitaciones- sino que las escuelas en sus constitución micropolítica (Foucault, 1980; Guattari y Suely, 2005; Ball, 2017) adopte una constitución participativa e inclusiva entre el profesorado mismo, ¿cómo construir escuelas inclusivas si el cuerpo docente es excluidos de la construcción del espacio educativo donde se despliegan? la escuela inclusiva se construye desde la práctica misma de la inclusión.

En la actualidad, la inclusión -usada como práctica discursiva- estaría produciendo cambios cosméticos dentro del sistema educativo, como es el trabajo colaborativo dentro del aula (Ministerio de Educación, 2017), donde el profesorado de enseñanza regular es acompañado por docentes diferenciales o profesionales de psicopedagogía para trabajar con nuevas metodologías y de esta manera todos puedan aprender dentro del aula, cambio cosmético ya que no se instala la necesidad de una formación docente que contemple que el profesorado en general sea especialista en atender a las diferencias, lo que implicaría una reestructuración de las subjetividades del futuro cuerpo docente del país. 
Las Necesidades Educativas Especiales son propuestas con la finalidad de eliminar las clasificaciones y etiquetas. No obstante, enfrenta el contrasentido que esta nominación es ya una etiqueta en sí misma, que solo cambia al significante para denominar y seguir categorizando a quienes presentan una condición distinta a lo que persigue una Sociedad del positivismo de lo normal (Diez, 2004; Han, 2017) a los estereotipos que establece la sociedad de la normalización (Foucault, 2001).

En nuestra experiencia docente ${ }^{7}$, esto queda evidente cuando se les nombra como estudiantes PIE -es decir, niños y/o niñas que presentan Necesidades Educativas Especiales y pertenecen al grupo del Programa de Integración Escolar-. Este aspecto es corroborado por las investigaciones de Diez (2004), López (2014) y López et al., (2014), donde sus hallazgos indican que se crea la categoría de "alumno integrado" o "niños PIE", que son víctimas de prácticas de estigmatización y segregación al interior de las escuelas y del sistema educativo. De esta forma, nos encontramos con un desplazamiento (Derrida, 1986) de sentido que podríamos atribuir a cierta dominación ideológica (Zizek, 2003).

Las formas en las que los sujetos que componen el Sistema educativo desarrollan el enactment de las políticas (Casimiro, 2016; Braun, Ball y Maguire, 2011) -cuya lógica parece más cercana a las eficiencias productivas empresariales que a criterios pedagógicosformativos, dada la fuerza que adquiere el New Public Management en educación (Morales, 2014)-, hace que se mantengan los procesos de construcción de escuelas inclusivas como un intento fallido.

\section{Conclusiones}

\subsection{Hacia una escuela inclusiva para la justicia social}

Para poder comprender el presente es siempre necesario mirar la historia, no sólo como historicidad sino como elemento constituyente del presente. En relación con los procesos integrativos, internacionalmente hablando, es a partir de las décadas del sesenta y setenta se comienza a hablar de integración con mayor fuerza en las escuelas. Esto queda demostrado en el informe Warnock (1978), donde se declara la importancia de entregar condiciones de igualdad de educación para niñas y niños, independiente de sus discapacidades, en los contextos del Sistema Educativo Británico. La importancia de este

\footnotetext{
7 Los investigadores somos profesoras y profesores (tres educadoras diferenciales y un profesor de filosofía con experiencia en escuelas especiales).
} 
informe radica en la influencia que significó para la producción de políticas integrativas en Chile, lo que queda demostrado al ser Fuente de referencia en los instructivos y sitios web que emana y mantiene el Ministerio de Educación (MINEDUC) chileno (Godoy, Meza, y Salazar, 2004).

Consideraciones respecto a las condiciones sociohistóricas de los sujetos como determinantes de un despliegue social equitativo, como si bastase solamente construir un sistema educativo que iguale los "puntos de partida" de unos y otros para conseguir una distribución social justa. Es decir que, mientras la práctica discursiva apela a una construcción social universalmente equitativa, en su praxis define la reducción del sujeto al contexto presente de su condición (necesitado de soportes educativos especiales), una univocidad relativa a su condición de discapacitado, de paso le ontologiza en esa condición.

El análisis de las trayectorias de los desplazamientos de sentidos entre integración a inclusión establece la performatividad de su uso, dado que, como tal, las prácticas discursivas tienden a establecer cierta realidad, a que la situación sea de esa manera. De esta forma, se habla de igualdad dentro de las políticas públicas, pero no se plantea como un deber y/o derecho para toda la población estudiantil, dejando entrever que seguimos inmersos dentro de una sociedad desigual que intenta la igualdad dentro del sistema educativo, como si las escuelas fuesen una burbuja dentro de las estructuras sociales. por esta razón apelamos a la necesaria imbricación que debe existir entre la noción de inclusión y la de justicia social, esto se observa en que "la distribución de las oportunidades educativas para asegurar dicho derecho no sería obligatoria ni universal" (Cifuentes, 2014, p. 6).

Los hallazgos sugieren que la liberación de la enseñanza impide la implementación de procesos inclusivos, ya que se puede inferir que dependerá de cada institución educativa el querer optar y ofrecer una educación de calidad dentro de sus establecimientos, siendo esto no un derecho de ley, sino que más bien una opción dependiendo de su proyecto educativo institucional y los requerimientos económicos de la institución o de su sostenedor. De esta forma, se puede señalar que no existe una clara justicia social, preferentemente relacionado con la participación del grupo estudiantil, obviando que, como tal, la justicia social "implica la promoción del acceso y la equidad para asegurar la plena participación en la vida social, especialmente para aquellos que han sido sistemáticamente excluidos" (Murillo y Hernández, 2011, p. 18).

Las lógicas de eficiencia que establece el new public management fortalecen las miradas evaluativas del sistema, conformando un tipo de evaluación de aprendizajes que 
promueve la segregación y la clasificación de estudiantes - pues centra su foco en el monitoreo de la gestión de los recursos y su relación con resultados; es decir, espera que la subvención que reciba el la población estudiantil sea eficientemente utilizada, respaldada y controlada- olvidando que dicha evaluación podría tener un abanico de posibilidades.

De la misma manera, se puede mencionar que, a pesar de que existieron desplazamientos en el significado tanto de la palabra integración como de inclusión, se denota un discurso político de hacernos creer que lo que se está realizando es inclusivo a pesar de que no lo es. Esto se demuestra dado que las políticas públicas proclamadas no son universales ni obligatorias y que lo que se ha realizado hasta hoy en las escuelas se encuentra muy lejano a inclusión. De este modo, a pesar de que la inclusión empieza a forma parte de lo que está aconteciendo en nuestro país, se puede indicar que todas las adecuaciones políticas que se han realizado se encuentran inmersas en la ambigüedad, dándole respuesta a las dudas que aparecen en torno a ellas en el mismo quehacer. Dicho de otro modo, los procesos inclusivos quedan plasmados en papel, reducida a los intentos de mediación del profesorado, reproduciendo éstos las construcciones sociales sobre la diversidad; pero, al momento de llevar a la práctica, se encuentran muy lejos de lograr los objetivos prometidos, quedando nuevamente la inclusión como un concepto inalcanzable a la realidad educativa, a pesar de que se encuentre promulgados en nuestras leyes chilenas. Los niveles de gestión de una educación inclusiva que persigue la justicia social se agotan en las tintas y nunca se derraman desde el papel hacia la praxis educacional.

Esta situación también se ve influenciada en que la gran mayoría de las acotaciones realizadas en las leyes y decretos no son vistas como una obligatoriedad y un derecho real para las personas con alguna discapacidad o necesidad educativa, lo que nuevamente provoca que queden en desmedro de oportunidades en relación con sus pares.

Las reformas políticas en torno a la educación inclusiva se encuentran directamente relacionadas con el tipo de sociedad en el cual estamos insertos, efectivamente la integración llegó para dar respuestas a muchos niños y niñas que presentaban necesidades educativas, donde en su momento ilusionó a muchos respecto a la igualdad de oportunidades dentro la educación. Sin embargo, con el tiempo se comenzó a visualizar una pseudo integración en los establecimientos educacionales, donde con el solo hecho de contar con la presencia de las personas ya bastaba para tomar como zanjado la práctica integrativa dentro de las salas de clases, como consecuencia no se lograron eliminar las barreras de aprendizajes que presenciaba el estudiantado con Necesidades Educativas 
Especiales, siendo por supuesto un intento fallido. Es decir, se actuó solo porque las políticas públicas lo pusieron como un tema importante a tratar en la educación y porque, además, somos un país que quiere demostrarse desarrollado a nivel internacional, pero que no reestructuró las culturas de los sujetos participantes del proceso educativo.

Al pasar el tiempo se ha comprobado que el modelo educativo integrador no ha logrado constituir escuelas acordes para aquello que ofertaba (Parra, 2010). Niños y niñas que, por contextos de género, etnia, circunstancias físicas han sido tradicionalmente excluidos y que en un momento pusieron todas las esperanzas y expectativas en este despliegue de normativas como La ley № 20.422 de Inclusión Social (2010) del Ministerio de Planificación de Chile, la Ley № 20.609 de No Discriminación (2012) del Ministerio Secretaría General de Gobierno, la Ley № 20.845 de Inclusión Escolar (2015) del Ministerio de Educación, y el Decreto N`83 (Ministerio de Educación, 2015a).

EI MINEDUC relata la promesa de la inclusión, y señala que ella debe formar parte de esta sociedad con la finalidad de alcanzar una educación de calidad para todos. Sin embargo, consideramos que la simple declaración de esto no es suficiente para construir una sociedad más equitativa y socialmente justa, ya que esta discurso tiene como correlato la adopción de una serie de tecnologías que abrazan la estandarización desde lo curricular (Aranda y Parra, 2014; Osandón et al., 2018) y de la estandarización del aprendizaje (Consejo Nacional de Educación, 2019) junto con la limitación de lo que se entiende por diversidad sólo aquello que entra en la clasificación clínica del Decreto № 170.

Según lo tratado anteriormente, se concluye que estamos en proceso de transición de la mirada y práctica de aceptar las diversidades en las aulas regulares, donde se reduce la problemática de construcción de sociedades equitativas a aspectos curriculares de la formación docente y esa búsqueda de perfiles de idoneidad, por lo tanto, queda mucho camino por recorrer y lograr que toda la población estudiantil pueda acceder a una educación pública que persiga los fines de la justicia social.

\section{Referencias}

Aranda, Virginia, y Parra, Hugo. (2014). Tiempos críticos y pedagogías críticas. Diálogos Educativos, 14(28), 119-134. Recuperado de http://www.dialogoseducativos.cl/revistas/n28/aranda

Ascorra, Paula., López, Verónica., y Urbina, Carolina. (2016). Participación estudiantil en escuelas chilenas con buena y mala convivencia escolar. Revista de Psicología, 25(2), 01-18. 
Ascorra, Paula., Carrasco, Claudia., López, Verónica., y Morales, Macarena. (2019). Políticas de convivencia escolar en tiempos de rendición de cuentas. Archivos Analíticos de Políticas Educativas, 27(31), 1-26.

Ball, Stephen. (2017). Subjectivity as a site of struggle: refusing neoliberalism? Subjectivity as a site of struggle: refusing neoliberalism? British Journal of Sociology of Education, 1129-1146. https://doi.org/10.1080/01425692.2015.1044072

Borri, Claudia. (2016). El movimiento estudiantil en Chile (2001-2014). La renovación de la educación como aliciente para el cambio político-social. Recuperado de https://webcache.googleusercontent.com/search?q=cache:uXQGFvRHMzkJ:https://rivist e.unimi.it/index.php/AMonline/article/download/7057/6905/+ycd=6yhl=esyct=clnkygl=cl

Braun, Annette., Ball, Stephen. J., y Maguire, Meg. (2011). Policy enactments in schools introduction: Towards a toolbox for theory and research. Discourse: Studies in the Cultural Politics of Education, 32(4), 581-583.

Casimiro, Alice. (2016). The theory of enactment by Stephen Ball: And what if the notion of discourse was different? Education Policy Analysis Archives, 24, 1-19.

CAST. (2008). Guía para el Diseño Universal del Aprendizaje. Wakefield: CAST. Recuperado de http://www.uco.es/aforac/media/recursos/Diseno_Universal_de_Aprendizaje.pdf

Chile, Ministerio de Educación de. (2015). Decreto Exento 83. Chile: Biblioteca del Congreso Nacional.

Cifuentes, Pamela., y Holz, Mauricio. (2014). Marco legal y reglamentario de la Educación Especial. Santiago, Chile: Biblioteca el Congreso Nacional de Chile Área de Políticas Sociales.

Consejo Nacional de Educación. (2019). Acuerdo No. 10, sobre estándares de aprendizaje. Santiago de Chile: El autor.

Diez, Adriana. (2004). Las "necesidades educativas especiales". Políticas educativas en torno a la alteridad. Cuadernos de Antropología Social, 19, 157-171.

Derrida, Jacques. (1986). De la gramatología. México DF., México: Siglo XXI.

Echeita, Gerardo. (2008). Inclusión y exclusión educativa. "Voz y quebranto". REICE. Revista Iberoamericana sobre Calidad, Eficacia y Cambio en Educación, 6(2), 9-18.

Falabella, Alejandra. (2015). El mercado escolar en Chile y el surgimiento de la nueva gestión pública: el tejido de la política entre la dictadura neoliberal y los gobiernos de la centroizquierda (1979 a 2009). Educação y Sociedade, 36(132), 699-722. https://doi.org/10.1590/es0101-73302015152420

Flick, U. (2004). Introducción a la Investigación Cualitativa. Madrid: Ediciones Morata. 
Foucault, Michael. (1980). Microfísica del poder. Madrid: La piqueta. Recuperado de http://www.pensamientopenal.com.ar/system/files/2014/12/doctrina39453.pdf

Foucault, Michael. (2001). Defender la sociedad. curso en el Collège de France (1975-1976). Buenos Aires: Fondo de Cultura Económica. Recuperado de https://www.uv.mx/tipmal/files/2016/10/M-FOUCAULT-DEFENDER-LA-SOCIEDAD.pdf

Fraser, Nancy. (1997). De la redistribución al reconocimiento?. Dilemas en tomo a la justicia en una época "postsocialista. Iustitia Interrupta: Reflexiones Críticas Desde La Posición "Postsocialista," 17-54.

Fraser, Nancy. (2008). La justicia social en la era de la política de identidad: redistribución, reconocimiento y participación. Revista de Trabajo, 4(6), 83-99.

García-Huidobro, Juan Eduardo., y Bellei, Cristián. (2006). La subvención escolar preferencial ¿remedio para la inequidad? Revista Mensaje, 547.

Glisczinski, Dan. (2018). Thematic Analysis. Journal of Transformative Education, 16(3), 175-175. https://doi.org/10.1177/1541344618777367

Godoy, María. P., Meza, María. L., y Salazar, Alida. (2004). Antecedentes históricos, presente y futuro de la educación especial en Chile. Recuperado de http://especial.mineduc.cl/wpcontent/uploads/sites/31/2016/08/201304151210180.doc Antecedentes Ed Especial.p $\underline{\mathrm{df}}$

Guattari, Félix., y Suely, Rolnik. (2005). Micropolíticas: cartográfias del deseo. Petropolis: Editora Vozes Ltda. Retrieved from https://www.traficantes.net/sites/default/files/pdfs/Micropolítica-TdS.pdf

Han, Byung-Chul. (2017). La expulsión de lo distinto. Barcelona: Herder Editorial.

Infante, Marta. (2010). Desafíos a la Formación Docente: Inclusión Educativa. Pontificia Universidad Católica de Chile, Facultad de Educación. Estudios Pedagógicos, 36(1), 287-297.

Inostroza, Fabián. A. (2015). Análisis crítico del discurso sobre la noción de estudiante con dificultades del aprendizaje en el marco de un Proyecto de Integración Escolar en una escuela municipal de Chile. Revista Mexicana de Investigación Educativa, 20(66), 941958.

Khan, Shamus. (2011). Privilege: The Making of an Adolescent Elite at St. Paul's School. Princeton, NJ: Princeton University Press.

López, Verónica. (2014). Discriminación y segregación: Efectos de la integración escolar sobre los proyectos de vida de estudiantes egresados de escuelas municipales que participaron en proyectos de integración escolar. Revista Latinoamericana de Inclusión Educativa, 8(2), 69-83. 
López, Verónica., Julio, Cristina., Pérez, María Victoria., Morales, Macarena., y Rojas, Carolina. (2014). Barreras Culturales para la Inclusión: Políticas y Prácticas de Integración en Chile. Revista de Educación, 363, 1-17.

Mayol, Alberto., y Azócar, Carla. (2011). Politización del malestar, movilización social y transformación ideológica: el caso "Chile 2011". Polis, 30, 1-22.

Ministerio de Educación de Chile. (1990). Normas para integrar alumnos discapacitados en establecimientos comunes, Decreto No. 490. Recuperado de https://www.leychile.cl/Navegar?idNorma=13743ybuscar=ds+490+1990

Ministerio de Educación de Chile. (1998). Normas para la integración social de personas con discapacidad, Decreto No. 1.2 Recuperado de https://www.leychile.cl/Navegar?idNorma=120356

Ministerio de Educación de Chile. (1999). Complementa decreto supremo de educación No 1, Decreto No. 374. Recuperado de http://especial.mineduc.cl/wpcontent/uploads/sites/31/2016/08/201304231731590.DecretoN374.pdf

Ministerio de Educación de Chile. (2005). Nuestro Compromiso con la Diversidad. Política nacional de Educación Especial. Santiago. Recuperado de http://especial.mineduc.cl/wp-content/uploads/sites/31/2016/08/POLiTICAEDUCESP.pdf

Ministerio de Educación de Chile. (2007). Ley de subvenciones a establecimientos educacionales y otros cuerpos legales, Pub. L. No. 20.201. Recuperado de https://www.leychile.cl/Navegar?idNorma=263059

Ministerio de Educación de Chile. (2008). Ley de subvención escolar preferencial, No. 20.248. Recuperado de https://www.leychile.cl/Navegar?idNorma=269001

Ministerio de Educación de Chile. (2009). Ley General de Educación, No. 20.370. Recuperado http://www.mineduc.cl/usuarios/convivencia escolar/doc/201103050142570.Ley N 20370 Ley General de Educacion.pdf

Ministerio de Educación de Chile. (2010). Fija normas para determinar los alumnos con NEE que serán beneficiarios de subvenciones para educación especial, Decreto No. 170. Recuperado de https://www.leychile.cl/N?i=1012570yf=2010-08-25yp=

Ministerio de Educación de Chile. (2013). Orientaciones Técnicas para Programas de Integración Escolar (PIE). Santiago, Chile: Gobierno de Chile.

Ministerio de Educación de Chile. (2015a). Decreto Exento 83. Recuperado de https://www.leychile.cl/Navegar?idNorma=162795

Ministerio de Educación de Chile. (2015b). Orientaciones para la revisión y actualización del Proyecto Educativo Institucional. Santiago, Chile: División de Educación General.

Ministerio de Educación de Chile. (2017). Manual de apoyo a sostenedores $y$ establecimientos educacionales, para la implementación del Programa de Integración Escolar (PIE), en el marco de la Inclusión. Santiago, Chile: Gobierno de Chile. 
Morales, Marjorie. (2014). New Public Management in Chile: Origins and Effects. Revista de Ciencia Política, 34(2), 427-438. Recuperado de https://scielo.conicyt.cl/pdf/revcipol/v34n2/art04.pdf

Murillo J., y Hernández R. (2011). Hacia un concepto de Justicia Social. Revista Iberoamericana sobre Calidad, Eficacia y Cambio en Educación, 9(4), 7-23.

Opertti, Renato., y Magni, Giorgia. (2018). Estado, mercado y currículum escolar: la experiencia chilena (1964-2018). (Cuestiones fundamentales y actuales del currículo, el aprendizaje y la evaluación No. 20). Recuperado de https://docplayer.es/95525956Estado-mercado-y-curriculum-escolar-la-experiencia-chilena.html

Osandón, Luis., Caro, Miguel., Magendzo, Abraham., Lavín, Sonia., González, Fabián y Cabaluz, Jorge. (2018). Estado, mercado y currículum escolar: la experiencia chilena (1964-2018). (Cuestiones fundamentales y actuales del currículo, el aprendizaje y la evaluación No. 20). Santiago. Recuperado de https://unesdoc.unesco.org/in/documentViewer.xhtml?id=p::usmarcdef 0000265296yfile=/in/rest/ annotationSVC/DownloadWatermarkedAttachment/attach import 3643dab6-c60d-4dc8-92aebee524b06e47\%3F \%3D265296spa.pdfylocale=esymulti=trueyark=/ark:/48223/pf000026529

Pairo, Fernando. (2011). Gabriel Salazar y María Olivia Monckeberg - La Educación en Chile. Recuperado de https://www.youtube.com/watch?v=VkXRrBFcRxw

Parra, Carlos. (2010). Educación Inclusiva: Un modelo de educación para todos. Revista ISEES, 8, 73-84.

Parra, Hugo. (2016). Discursos teoréticos postmodernos en la diversificación curricular (Tesis de Magister). Universidad Metropolitana de Ciencias de la Educación, Santiago, Chile.

Peña, Mónica. (2013). Análisis crítico de discurso del decreto 170 de subvención diferenciada para necesidades educativas especiales: El diagnóstico como herramienta de gestión. Psicoperspectivas, 12(2), 9-103

Piketty, Thomas. (2013). Le capital au XXle siècle. París, Francia: Le Seuil.

República de Chile. (2009), Ley 20.370 o Ley General de Educación. Recuperado de http://www.mineduc.cl/usuarios/convivencia_escolar/doc/201103050142570.Ley_N_203 70_Ley_General_de_Educacion.pdf

Reyes-Jedlicki, L., Miranda, C., Santa Cruz, E., Cornejo, R., Núñez, M., Arévalo, A., y Hidalgo, F. (2014). ubjetividades y saberes docentes en el sistema educativo chileno: un análisis desde las concepciones de formadores de profesores. Estudios Pedagógicos, 2(40), 183-203. https://doi.org/https://dx.doi.org/10.4067/S0718-07052014000200011

Ricœur, Paul. (2000). Narratividad, fenomenología y hermenéutica. Lecturas y Conversaciones Con Paul Ricoeur, 189-207.

Ritchie, Jane., and Lewis, Jane. (2003). The Foundations of Qualitative Research. Qualitative research practice: $A$ guide for social science students and researchers. London: Sage. https://doi.org/10.4135/9781452230108 
Sandoval, Casilimas. (2002). Investigación Cualitativa. Bogotá: Instituto colombiano para el Fomento de la Educación Superior.

Silva, Beatriz. (2019). La "Revolución Pingüina" y el cambio cultural en Chile. Recuperado de https://www.researchgate.net/publication/338051589 La Revolucion Pinguina y el Ca mbio Cultural en Chile/link/5dfbc0da92851c83648aed90/download

Skliar, Carlos. (2002). Alteridades y pedagogías. O... ¿y si el otro no estuviera ahí? Educação y Sociedade, 23(79), 85-123.

Skliar, Carlos. (2014). Seminario Internacional de Educación Inclusiva y Tecnologías de la Comunicación [video]. Recuperado de https://www.youtube.com/watch?v=G1vxLffM1ZY

Valverde, Sergio. (2003). Paul Ricoeur: Hermenéutica. Revista de Filosofía, 2(104), 51-59.

Verger, Antoni., Moschetti, Mauro. y Fontdevila, Clara. (2017). La privatización educativa en América Latina. Una cartografía de políticas tendencias y trayectorias. Barcelona: Internacional de la Educación. Recuperado de https://download.eiie.org/Docs/WebDepot/Privatizacion 1-Abril.pdf

Warnock, M. (1978). Report of the Committee of Enquiry into the Education of Handicapped Children and Young People. London, England: HMSO.

Zizek, Slavoj. (2003). El sublime objeto de la ideología. Buenos Aires, Argentina: Siglo XXI. 
Revista indizada en
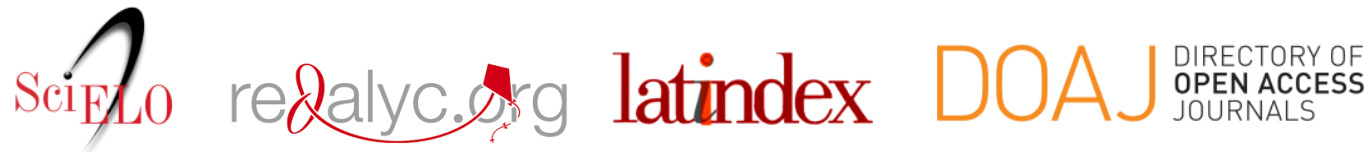

Distribuida en las bases de datos:

๑ Dialnet

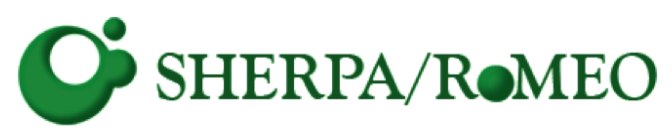

REDIB

Red Iberoamericana

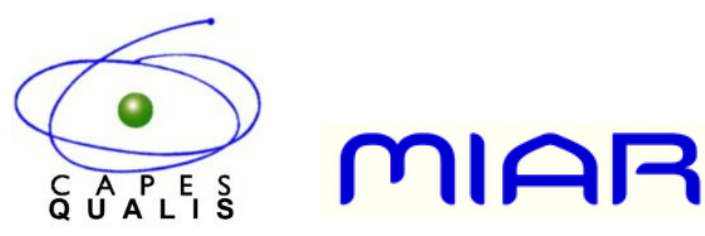

